\title{
Periodic Artifact Reduction in Fourier Transforms of Full Field Atomic Resolution Images
}

\author{
Robert Hovden ${ }^{1}$, Yi Jiang ${ }^{2}$, Huolin L. Xin ${ }^{3}$ and Lena F. Kourkoutis ${ }^{1,4}$ \\ ${ }^{1}$ School of Applied \& Engineering Physics, Cornell University, Ithaca, NY, USA. \\ ${ }^{2}$ Department of Physics, Cornell University, Ithaca, NY, USA. \\ ${ }^{3}$ Center for Function Nanomaterials, Brookhaven National Laboratory, Upton, NY, USA. \\ ${ }^{4}$ Kavli Institute at Cornell for Nanoscale Science, Ithaca, NY, USA.
}

Modern electron microscopists have the luxury of studying the atomic structure of materials directly through real space imaging. With sub-Angstrom resolution that distinguishes atomic columns in a crystal, one can count the atoms across a grain boundary, identify the structure of interfaces, and locate individual defects and dopants. However, real-space analysis of crystals is not always sufficient and the discrete Fourier transform (DFT) of atomic resolution images is routinely used to mimic a diffraction pattern-with reciprocal lattice spots reflecting the symmetry and spacing of a specimen's crystal structure. However, when calculating a Fourier transform, periodic boundary conditions are imposed and sharp discontinuities between the edges of an image cause a cross-patterned artifact along the reciprocal space axes (seen in Fig. 1d).

Traditionally, these DFT artifacts are reduced by applying a damping window to the real space image. However, windowing is not a universal approach - the window function can influence the resolution, shape, and intensity of spots in Fourier space [1]. More critically, by weighting the real space image the center of the window is overrepresented and important features toward the edges of the field of view can be missed. Figure 2 shows a $\mathrm{LaVO}_{3} / \mathrm{SrTiO}_{3}$ multilayer with one layer exhibiting a super-lattice structure. From the real-space image (Fig. 2a), this super-lattice is difficult to detect, but it clearly appears as additional peaks in the Fourier transform (Fig. 2d). In the windowed transform (Fig. 2e), however, this structure would be missed entirely_even when applying the relatively large Hann window (Fig. 2b).

Here we demonstrate that the recently developed Periodic Plus Smooth Decomposition $(\mathrm{P}+\mathrm{S})$ technique provides a simple, efficient method for reliable removal of artifacts caused by edge discontinuities in atomic resolution S/TEM images (Fig. 1) [2]. In this method, edge discontinuities are reduced by subtracting a smooth background determined by solving Poisson's equation with boundary conditions set by the image edges [3]. The resulting Fourier transform reflects the original image's DFT, but without the cross pattern artifact (Fig. 1e). Unlike windowed Fourier transforms, P+S Decomposition maintains sharp reciprocal lattice peaks and uses all information from the image's field of view-shown in Fig. 2f, the super-lattice peaks are preserved. This approach provides an accurate estimation of the spectrum in regions where reciprocal lattice peaks occur. Since the Fourier transform is a linear operator, the DFT of the smooth component (Fig. 1f) shows the exact intensity removed from the unprocessed DFT.

In a field where Fourier transforms are among the most common analysis tools, $\mathrm{P}+\mathrm{S}$ Decomposition can provide substantial utility for aberration-corrected S/TEM. [4]

[1] F.J. Harris, Proc IEEE 66, 51-83 (1978).

[2] R. Hovden et. al, Microsc. Microanal., doi:10.1017/S1431927614014639 (2015).

[3] L. Moisan, J. Math Imaging Visualization, 39 161-179 (2013).

[4] This work used the CCMR EM facility supported by NSF MRSEC program (DMR-1120296). 

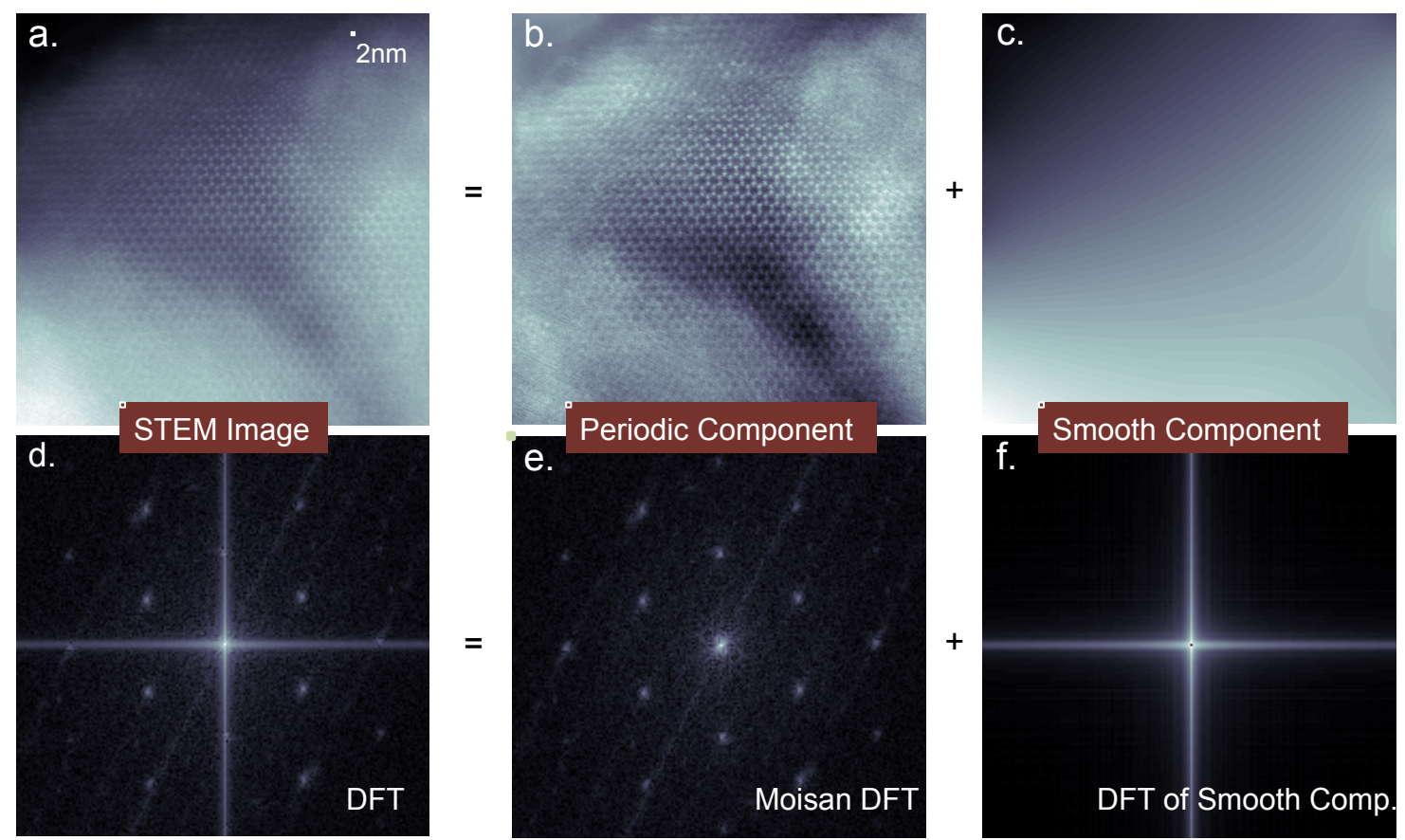

Figure 1. $\mathrm{P}+\mathrm{S}$ Decomposition of CdSe [111] STEM image. Discontinuity in the periodic boundaries manifest as cross pattern artifact in the DFT (d). The P+S Decomposition creates periodic boundary continuity (b) by removing a smooth background component (c) from the image. This removes the edge artifacts (e). The background component shows exactly what has been removed (f) to create the artifact free DFT (e). DFT's are log-absolute; scan direction is $26.5^{\circ}$ clockwise from vertical.

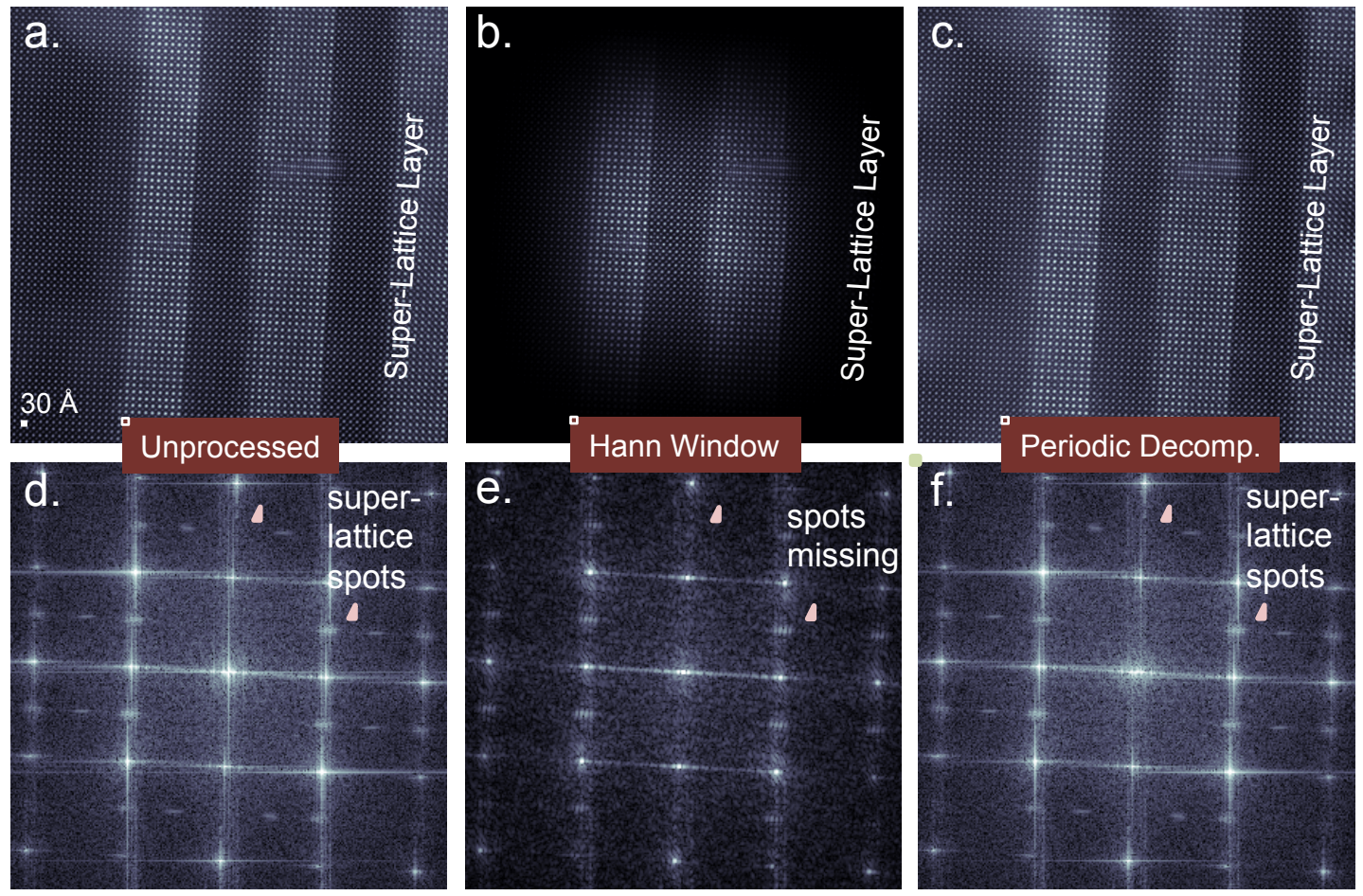

Figure 2. $\mathrm{LaVO}_{3} / \mathrm{SrTiO}_{3}$ with one layer showing a super-lattice structure (a) that appears as additional peaks in the DFT (d). A windowed DFT heavily weights the center of the image (b) and consequently misses the super-lattice peaks (e). P+S Decomposition utilizes the entire field of view (c), preserving the atomic superlattice structure in the DFT (f). Sample grown by the group of H.Y. Hwang (Stanford University). 